\title{
BMJ Open Protocol for a pragmatic randomised controlled trial of Body Brain Life - General Practice and a Lifestyle Modification Programme to decrease dementia risk exposure in a primary care setting
}

\author{
Sarang Kim, ${ }^{1}$ Mitchell McMaster, ${ }^{1}$ Susan Torres, ${ }^{2}$ Kay L Cox, ${ }^{3}$ \\ Nicola Lautenschlager, ${ }^{4}$ George W Rebok, ${ }^{5}$ Dimity Pond, ${ }^{6}$ Catherine D'Este, ${ }^{7}$ \\ Ian McRae, ${ }^{1}$ Nicolas Cherbuin, ${ }^{1}$ Kaarin J Anstey ${ }^{1,8,9}$
}

To cite: Kim S, McMaster M, Torres S, et al. Protocol for a pragmatic randomised controlled trial of Body Brain Life-General Practice and a Lifestyle Modification Programme to decrease dementia risk exposure in a primary care setting. BMJ Open 2018;8:e019329. doi:10.1136/ bmjopen-2017-019329

- Prepublication history and additional material for this paper are available online. To view these files, please visit the journal online (http://dx.doi. org/10.1136/bmjopen-2017019329).

Received 29 August 2017 Revised 9 February 2018 Accepted 14 February 2018

Check for updates

For numbered affiliations see end of article.

Correspondence to

Dr Sarang Kim;

sarang.kim@anu.edu.au

\section{ABSTRACT}

Introduction It has been estimated that a 10\%-25\% reduction in seven key risk factors could potentially prevent 1.1-3.0 million Alzheimer's disease cases globally. In addition, as dementia is preceded by more subtle cognitive deficits which have substantial social and economic impact, effective preventative interventions would likely have more extensive benefits. The current study evaluates in primary care a multidomain riskreduction intervention targeting adults with high risk of developing dementia.

Methods and analysis A randomised controlled trial (RCT) is being conducted to evaluate three intervention programmes using a pragmatic approach suitable to the clinic: (1) a 12-week online and face-to-face dementia risk-reduction intervention (Body Brain Life-General Practice (BBL-GP)); (2) a 6-week face-to-face group lifestyle modification programme (LMP); and (3) a 12week email-only programme providing general health information. We aim to recruit 240 participants, aged 18 and over, to undergo a comprehensive cognitive and physical assessment at baseline and follow-ups (postintervention, 18, 36 and 62 weeks). The primary outcome is dementia risk measured with the modified version of the Australian National University—Alzheimer's Disease Risk Index Short Form. Secondary outcomes are cognitive function measured with Trails $A$ and $B$, and the Digit Symbol Modalities Test; physical activity with moderate-vigorous physical activity and the International Physical Activity Questionnaire; depression with the Centre for Epidemiological Studies Depression; cost evaluation with the 12-item Short Form Health Survey, Framingham Coronary Heart Disease Risk Score and Australian Type 2 Diabetes Risk Assessment Tool; diet quality with the Australian Recommended Food Score; and sleep quality with the Pittsburgh Sleep Quality Index.

Ethics and dissemination This RCT is a novel pragmatic intervention applied in a primary care setting to reduce the dementia risk exposure in adults at high risk. If successful, BBL-GP and LMP will provide a versatile, evidence-
Strengths and limitations of this study

- Body Brain Life-General Practice Programme has been built on our dementia prevention research programme which has been shown to reduce cognitive decline in older adults at risk of dementia.

- This pragmatic trial evaluates a multidomain risk-reduction intervention in primary care targeting adults at increased risk of developing dementia.

- A naturalistic approach ensures the programme can be adapted efficiently in primary care settings if proven effective.

- We are aware that most of our outcomes are self-reported and therefore can be subjective. Accordingly, we will interpret data conservatively.

based package that can be easily and quickly rolled out to other primary care settings and which can be scaled up at relatively low cost compared with other strategies involving intensive interventions.

Trial registration number ACTRN12616000868482

\section{INTRODUCTION}

No cure is available for Alzheimer's disease (AD) and other types of dementia. However, it is estimated that an achievable $10 \%-25 \%$ reduction in seven key risk factors could prevent 1.1-3.0 million AD cases internationally. ${ }^{1}$ It is also estimated that if each of the seven risk factors was to be reduced by $5 \%$, $10 \%, 15 \%$ and $20 \%$ per decade, dementia prevalence would be reduced by between $1.6 \%$ and $7.2 \%$ in $2020,3.3 \%$ and $14.9 \%$ in $2030,4.9 \%$ and $22.8 \%$ in 2040 and $6.6 \%$ and $30.7 \%$ in $2050 .{ }^{2}$ Furthermore, as dementia is preceded by more subtle cognitive deficits 
which have substantial social and economic impacts, effective preventative interventions would likely have additional benefits.

Increasingly and to afford a greater chance of producing detectable changes during study timeframes, the dementia research community has focused on multidomain interventions that address multiple risk factors simultaneously. ${ }^{3}$ Among individuals with high risk factor burden, cognitive decline can be reduced (and possibly reversed) as a result of cardiovascular risk reduction and increase in activities that stimulate and protect the brain including cognitive, social and physical activity (PA) and an appropriate diet. ${ }^{4} \mathrm{AD}$ and cardiovascular disease share cardiometabolic and lifestyle risk factors and cardiovascular risk reduction can be achieved by smoking cessation, increasing physical activities, adopting a healthy diet, reducing abnormally high blood pressure and cholesterol in mid-life, and managing major depression, overweight/obesity in mid-life and diabetes if present. ${ }^{5}$ Altogether, the literature supports the view that multidomain interventions aimed at reducing cardiometabolic risk and promoting behaviours shown to protect against dementia will contribute to preventing cognitive decline, reduce overall risk of $\mathrm{AD}$ and lower depressive symptoms.

Insufficient PA is the risk factor with the most evidence to support its role as a treatment for mild cognitive impairment ${ }^{6}$ and more generally cognitive decline. ${ }^{7} \mathrm{PA}$ has also been shown in randomised controlled trials (RCTs) to benefit several other risk factors for dementia including depression, ${ }^{8}$ and cardiovascular risk factors. ${ }^{9}$ PA modifies multiple risk factors and it has direct benefits for brain health and cognition.

To bring about risk reduction, there needs to be longlasting behavioural change in multiple areas. Achieving this requires using techniques such as goal setting, decreasing barriers to change, improving self-monitoring, having access to information and maintaining motivation. ${ }^{10} 11$ Therefore, this RCT investigates whether Lifestyle Management Programmes that offer health-promoting information and practical behaviour change techniques that can be implemented in daily life can reduce dementia risk.

\section{Recruitment in general practice setting}

Primary care is an ideal setting for the implementation of the current programme because it is where adults with high risk of developing dementia are identified and early intervention and treatment are provided. ${ }^{12}$ Assessment of cardiovascular risk factors is common in primary care, as is advice about PA and diet. General practitioners (GP) commonly screen for diabetes and increasingly identify depression. GPs are often the first point of contact for patients who are worried that they may have dementia. ${ }^{13}$

Although there has been one study conducted in primary care setting with elderly participants $(70-78$ years old) addressing cardiovascular risk factors, ${ }^{14}$ the current programme is the first of its kind to provide interventions to adults (18 years and above) at the primary care setting, addressing both cardiovascular and lifestyle risk factors of dementia.

\section{METHODS AND ANALYSIS \\ Study setting and design}

This project is a 6-12weeks, pragmatic single-blind RCT that is designed to assist participants develop and maintain a healthy lifestyle, as well as manage chronic diseases. The study evaluates the implementation of an evidence-based dementia risk-reduction programme that we developed and have evaluated previously on volunteers ${ }^{15}$ and which has now been adapted for primary care (Body Brain Life-General Practice (BBL-GP)). The primary care setting in which the study is held already conducted a Lifestyle Modification Programme (LMP) aimed at helping to manage chronic disease and maintaining a healthy lifestyle. The LMP was initially developed to consist of 12 sessions over 12 weeks. However, its format was changed prior to this trial to have 12 sessions over 6 weeks. This decision was made by the clinic which provides this programme in order for the programme to be offered four times a year. The current LMP was chosen as a comparison condition for feasibility and to enable evaluation of an existing programme for dementia and cardiovascular risk reduction. The efficacy of the existing LMP had not previously been evaluated.

The existing LMP included 6 weeks of face-to-face group education sessions. The BBL-GP Programme included 12 weeks of individually tailored online education sessions with 1-hour face-to-face individual sessions with a dietitian and an exercise physiologist. The BBL-GP and LMP are being compared with an active control group receiving weekly email with links to health information. The study is being conducted in Canberra, Australian Capital Territory, Australia. The trial has been designed and is conducted according to the Consolidated Standards of Reporting Trials statements for non-pharmaceutical ${ }^{16}$ and pragmatic ${ }^{17}$ trials and is reported according to the Standard Protocol Items: Recommendations for Interventional Trials guidelines. ${ }^{18}$

\section{Participants}

Participants are being recruited from the National Health Co-op (NHC), the largest bulk billing general practice organisation in Canberra comprising eight clinics. Bulk billing is a payment option where the doctor bills directly the universal health insurance system (Medicare) in Australia for a medical service that the patients receive. Invitation emails have been sent to all members excluding members who are inactive (those who did not renew their memberships), those aged less than 18 years or without email addresses. Posters at the clinics are also being used for the recruitment. Potential participants who express their interest by contacting the LMP coordinator at the NHC or registering on the NHC's website are assessed against the inclusion and exclusion criteria. These are the types of adults who a GP would refer to a 
dementia risk-reduction trial in 'real life' and on whom we are aiming to evaluate our intervention in a naturalistic context. In addition, risk factors for dementia exert their influence over decades and thus the earlier one decreases their risk exposure, the more impact it is likely to have over their lifespan. Therefore, this intervention programme is open to anyone aged 18 and older. If criteria are met, information sheets and consent forms are sent to potential participants. On return of consent forms via email, each participant is officially registered to the study and allocated a unique identity number as well as an online account. Recruitment began in July 2016 for the duration of 13 months.

\section{Inclusion criteria}

A naturalistic approach is used in recruitment and the study inclusion criteria being used for this study are those already used by the NHC to refer patients to the LMP (prior to this research project). The inclusion criteria are pragmatic as the practice already had criteria for referral to their LMP and in developing the protocol, it became clear that introducing a second set of inclusion criteria would make implementation difficult and reduce participant numbers. We therefore decided to use the principle that if a GP would refer the patient to the LMP, then they would be eligible for the trial. This is a pragmatic feature of the current trial that significantly differs from our original BBL trial. We aimed to optimise the seamlessness of the intervention in primary care and use existing referral pathways to increase the probability that the intervention is conducted in a manner that could lead to implementation in real life. Participants must be aged 18 years and over, reside in the Australian Capital Territory, be current financial members of the NHC, have access to a computer and internet connection at home, be fluent in English, Australian permanent residents or citizens (for bulk billing eligibility) and must be the only person in their household who is taking part in this study to prevent being randomly assigned to different groups and sharing information about their interventions with each other received. To be eligible for the study, participants are also required to have a chronic health condition (high blood pressure, heart disease, type 2 diabetes or 'prediabetes', osteoporosis, osteoarthritis, polycystic ovary syndrome, kidney or liver disease and depression/anxiety) or be overweight or obese (body mass index $>25$ ). They are also required to agree to commit 1-2 hours a week to complete the programme and be interested in obtaining advice on improving their lifestyle to reduce the risk of or better manage chronic disease. Participants are required to complete online assessments and attend NHC at baseline and 18, 36 and 62 weeks after the commencement of the intervention for medical and cognitive assessments.

\section{Exclusion criteria}

Participants are not eligible to enrol in the trial if they have significant and unstable medical and psychiatric conditions that would prevent participation in the trial.
They are also ineligible if they have sensory deficits or mobility limitations that would prevent or substantially restrict the delivery of the assessment or intervention, have cognitive impairment or are pregnant. Those who have previously participated in the LMP were excluded from participation. However, those who may be/have been participating in other trials, unknown to authors, were not excluded.

\section{Sample size calculations}

Sample size calculations were estimated using $\mathrm{G}^{*}$ Power (V.3.1.9.2; http://www.gpower.hhu.de/en.html) and have been based on medium effect size as observed in the previous Body Brain Life (BBL) project with the same primary outcome. ${ }^{15}$ To detect a medium effect $(0.5 \mathrm{SD})$ in a 3-group design (1:1:1), four measurements with a $5 \%$ risk of type 1 error $(\alpha)$ and $80 \%$ power, a total sample size of 159 persons is required. To account for a $33 \%$ attrition (based on previous Lifestyle Modification Programme by NHC using the same inclusion and exclusion criteria and targeting the same age group), a baseline sample of 240 is being recruited (80 in BBL-GP group, 80 in LMP group and 80 in control group).

\section{Assessments}

Participants who meet all inclusion and no exclusion criteria are invited to complete online surveys and visit NHC for the baseline evaluation, and for weeks 18, 36 and 62 follow-ups. Immediate follow-up is also conducted online at week 7 for LMP, and week 13 for BBL-GP and control groups. Table 1 summarises the assessment measures and schedule.

\section{Screening measures and covariate}

In addition to the above inclusion and exclusion criteria, further screening measures are conducted to ensure that participants are capable of taking part in the study. The Adult Pre-exercise Screening System (APSS) ${ }^{19}$ is used at the baseline assessment to identify individuals with acuterisk/high-risk conditions, or who may be at higher risk of an adverse event during exercise. To screen for any cognitive impairment $(<25)$, the Mini-Mental State Examination $^{20}$ is administered to participants aged 60 and older.

Health efficacy and motivation for healthiness subscales from the Multidimensional Health Questionnaire ${ }^{21}$ are used to measure the extent to which people believe they have the ability, capability, skills and talents to take care of their own physical health, and to measure people's motivation to keep in good physical health.

\section{Primary outcome}

The primary outcome is one's exposure profile to demonstrated risk factors for AD. It is measured with a modified version of the Australian National University Alzheimer's Disease Risk Index-Short form (ANU-ADRI-SF). ${ }^{22}$ The ANU-ADRI-SF comprises validated scales assessing 15 individual risk and protective factors for $\mathrm{AD}$ and dementia. Intraclass correlation coefficients suggested that the reliability of the ANU-ADRI-SF compared with the original 
Table 1 Assessment measures at the baseline and follow-up evaluations

Immediate follow-up (week 7 for

LMP and week 13 for BBL-GP

\begin{tabular}{llllll} 
Assessment measure & Baseline & and active control group) & Week 18 & Week 36 & Week 62 \\
\hline Screening & & & & & \\
APSS & $\sqrt{ }$ & & & \\
MMSE (if 60+) & $\sqrt{ }$ & &
\end{tabular}

Questionnaires

$\begin{array}{llllll}\text { ANU-ADRI } & \sqrt{ } & \sqrt{ } & \sqrt{ } & \sqrt{ } & \sqrt{ } \\ \text { PSQI } & \sqrt{ } & \sqrt{ } & \sqrt{ } & \sqrt{ } & \sqrt{ } \\ \text { ARFS } & \sqrt{ } & \sqrt{ } & \sqrt{ } & \sqrt{ } & \sqrt{ } \\ \text { SF-12 } & \sqrt{ } & \sqrt{ } & \sqrt{ } & \sqrt{ } & \sqrt{ } \\ \text { MHQ } & \sqrt{ } & & & & \end{array}$

Cognitive measures

\begin{tabular}{|c|c|c|c|c|}
\hline Trails $A+B$ & $\sqrt{ }$ & $\sqrt{ }$ & $\sqrt{ }$ & $\sqrt{ }$ \\
\hline DSMT & $\sqrt{ }$ & $\sqrt{ }$ & $\sqrt{ }$ & $\sqrt{ }$ \\
\hline \multicolumn{5}{|c|}{$\begin{array}{l}\text { Physical and medical evaluation (by } \\
\text { doctors and nurses) }\end{array}$} \\
\hline MVPA & $\sqrt{ }$ & $\sqrt{ }$ & $\sqrt{ }$ & $\sqrt{ }$ \\
\hline Blood pressure & $\sqrt{ }$ & $\sqrt{ }$ & $\sqrt{ }$ & $\sqrt{ }$ \\
\hline Height, cm & $\sqrt{ }$ & & & \\
\hline Weight, kg & $\sqrt{ }$ & $\sqrt{ }$ & $\sqrt{ }$ & $\sqrt{ }$ \\
\hline Waist and hip, cm & $\sqrt{ }$ & $\sqrt{ }$ & $\sqrt{ }$ & $\sqrt{ }$ \\
\hline Body composition & $\sqrt{ }$ & $\sqrt{ }$ & $\sqrt{ }$ & $\sqrt{ }$ \\
\hline Framingham CHD & $\sqrt{ }$ & $\sqrt{ }$ & $\sqrt{ }$ & $\sqrt{ }$ \\
\hline AUSDRISK & $\sqrt{ }$ & $\sqrt{ }$ & $\sqrt{ }$ & $\sqrt{ }$ \\
\hline
\end{tabular}

ANU-ADRI, Australian National University-Alzheimer's Disease Risk Index; APSS, Adults Pre-exercise Screening System; ARFS, Australian Recommended Food Score; AUSDRISK, Australian Type 2 Diabetes Risk Assessment Tool; BBL-GP, Body Brain Life-General Practice; DSMT, Digit Symbol Modalities Test; Framingham CHD, Framingham Coronary Heart Disease Risk Score; LMP, Lifestyle Modification Programme; MHQ, Multidimensional Health Questionnaire; MMSE, Mini-Mental State Examination; MVPA, Moderate-vigorous physical activity; PSQI, Pittsburgh Sleep Quality Index; SF-12, 12-item Short Form Health Survey.

ANU-ADRI was moderate to strong (0.77-0.99) and statistically significant $(\mathrm{p}<0.001)$ except for cognitive activity. Therefore, for the assessment of engagement in cognitive activities levels only, items from the original ANU-ADRI ${ }^{2324}$ are used in place of those from the ANU-ADRI-SF due to limitations of the latter.

\section{Secondary outcomes}

Secondary outcomes include cognitive function, PA level, depressive symptoms, cost of interventions, diet and sleep quality. They are measured as follows: cognitive function is assessed with processing speed, task switching and executive function using Trails $\mathrm{A}$ and $\mathrm{B}$, and the Digit Symbol Modalities Test (DSMT). These tests were chosen because the executive function is the most sensitive cognitive domain to PA interventions ${ }^{25}$ and a decline in processing speed is associated with cardiovascular risk factors. ${ }^{26}$ Both Trails and DSMT have been used widely and have been reported to have good reliability and validity. ${ }^{27-30}$ Moderate-vigorous physical activity (MVPA) is a continuous measure of activity that registers three or more metabolic equivalents for $10 \mathrm{~min}$ or longer on an ActiGraph Link activity monitor (http://actigraphcorp. $\mathrm{com} /$ products/actigraph-link/), which is worn for 7 days. Self-reported PA is also being recorded using the short form of the International Physical Activity Questionnaire (IPAQ), ${ }^{31}$ which is part of the ANU-ADRI-SF. Reliability and validity of IPAQ have been tested and confirmed across 12 countries. ${ }^{31}$ Depression is being assessed with the Centre for Epidemiological Studies Depression (CES-D) Scale, ${ }^{32}$ which is also part of the ANU-ADRI. CES-D Scale has a very high internal consistency and validity. ${ }^{32}$

Health outcomes are assessed with the 12-item Short Form (SF-12) Health Survey, ${ }^{33}$ Framingham Coronary Heart Disease (CHD) Risk Score ${ }^{34}$ and Australian Type 2 Diabetes Risk Assessment Tool (AUSDRISK) ${ }^{35}$ to enable cost-effectiveness evaluation of the two health promotion interventions. SF-12 measures both physical and mental health status and has acceptable validity and reliability. ${ }^{36}{ }^{37}$ Framingham CHD is a validated tool to assess cardiovascular diseases ${ }^{38}$ and AUSDRISK is a diabetes 
Table 2 Comparison of intervention programmes

\begin{tabular}{|c|c|c|c|}
\hline & LMP & BBL-GP & Active control \\
\hline Previously applied: & $\begin{array}{l}\text { Yes, in primary care. } \\
\text { Evaluation has not been } \\
\text { carried out. }\end{array}$ & $\begin{array}{l}\text { Yes, with member of general public } \\
\text { with concern about developing } \\
\text { dementia. Never been tested in } \\
\text { primary care setting. }\end{array}$ & $\begin{array}{l}\text { Yes, with member of general public } \\
\text { with concern about developing } \\
\text { dementia. }\end{array}$ \\
\hline Duration & 6 weeks & 12 weeks & 12 weeks \\
\hline Frequency & Weekly & Weekly & Weekly \\
\hline Format & $\begin{array}{l}\text { Face-to-face group } \\
\text { sessions }\end{array}$ & $\begin{array}{l}\text { 1-hour individual session with } \\
\text { dietitian, 1-hour individual session } \\
\text { with exercise physiologist, eight } \\
\text { online modules }\end{array}$ & $\begin{array}{l}\text { Weekly emails containing health } \\
\text { information such as health status of } \\
\text { Australians, PA and nutrition, alcohol } \\
\text { and tobacco, and mental health. }\end{array}$ \\
\hline
\end{tabular}

BBL-GP, Body Brain Life-General Practice; LMP, Lifestyle Modification Programme; PA, physical activity.

risk assessment tool based on demographic, lifestyle and simple anthropometric measures. ${ }^{35}$

Dietary quality is assessed with a food-based diet quality index, the Australian Recommended Food Score

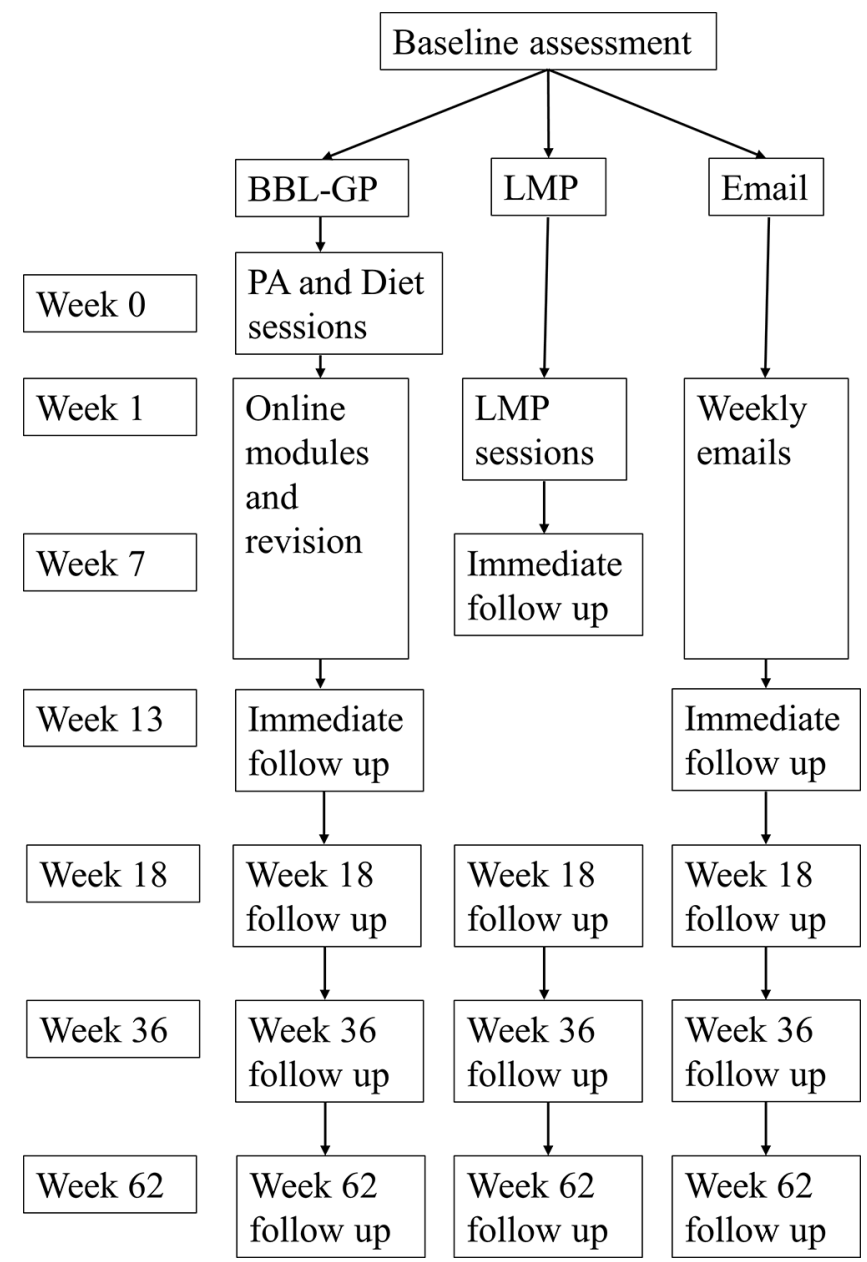

Figure 1 Study flow chart. BBL-GP, Body Brain LifeGeneral Practice; LMP, Lifestyle Modification Programme; PA, physical activity.
(ARFS). ${ }^{39}$ The ARFS is aligned with Australian Dietary Guidelines $^{40}$ and the Australian Guide to Healthy Eating ${ }^{41}$ recommendations. The ARFS total ranges from 0 to 73 and includes eight subscales: vegetables $(0-21)$, fruit $(0-12)$, protein $(0-7)$, vegetarian alternatives $(0-6)$, grains (0-13), dairy (0-11), water (0-1), sauces and condiments (0-2). Higher scores indicate greater compliance with the Australian Dietary Guidelines and therefore better diet quality. The ARFS has demonstrated good validity and reproducibility. ${ }^{39}$

Lastly, the Pittsburgh Sleep Quality Index (PSQI) ${ }^{42}$ is an effective instrument used to measure the quality and patterns of sleep in adults. It differentiates 'poor' from 'good' sleep quality by measuring seven areas (components): subjective sleep quality, sleep latency, sleep duration, habitual sleep efficiency, sleep disturbances, use of sleeping medications and daytime dysfunction over the last month. PSQI reports great test-retest reliability $(0.87)$ and high correlations with sleep log data. ${ }^{43}$

\section{Randomisation}

On completion of the baseline assessment, participants are randomly allocated into one of the three groups (see table 2 and figure 1). The allocation sequence is computer generated by an independent researcher and is not known to the study team at the time of enrolment and baseline assessment. A permuted block randomisation sequence comprising block sizes of six stratified by gender and age group (18-49 vs 50+) is used. The project manager who is not involved with conducting assessments assigns the participants into groups according to the generated sequence and notifies participants of their group allocation via email.

\section{Interventions}

Group 1: BBL-GP

BBL-GP is an intervention package that builds on our dementia prevention research programmes. This includes 
an online dementia risk-reduction programme called the BBL (trial ID: ACTRN12612000147886) ${ }^{1544}$ and the Fitness for the Ageing Brain Study (trial ID: ACTRN 12609000755235). ${ }^{45}{ }^{46}$ Contents for the BBL-GP online modules have been revised after extensive consumer evaluation by members of the Dementia Australia (formally known as Alzheimer's Australia) Consumer Dementia Research Network as well as members of the public and from participant feedback after the previous trial. The PA programme has also been modified for a younger age group to 18 years and older as previous programmes targeted middle aged and older adults. The ActiGraph device was introduced to measure the objective amount and intensity of PA. This revised programme (Body Brain Life-Fit) was piloted with the general public (trial ID: ACTRN12615000822583; manuscript in preparation).

Participants in the BBL-GP group are required to complete eight modules (dementia literacy, risk factors, PA, nutrition, health, cognitive activity, social activity and mood) delivered online. Prior to commencing online modules, participants in the BBL-GP group also receive an individually tailored plan/programme for both dietary and PA interventions developed and delivered by a dietitian and an exercise physiologist, respectively, during a face-to-face assessment. This is to ensure the dietary prescription and level of PA are suitable and tailored to individual participants.

\section{PA session}

The session duration and frequency of the PA programme varies between participants based on baseline PA levels and individual tailoring. An exercise physiologist designs an individual programme for the participant, delivers this in a face-to-face workshop and monitors the PA programme via the returned diaries and telephone monitoring. For those not doing any regular PA at baseline, the target is $150 \mathrm{~min} /$ week moderate intensity PA, with moderate intensity defined as a score of 10-12 on the Borg Rating of Perceived Exertion Scale (RPE) ${ }^{47}$ For those who are doing regular PA but for less than $100 \mathrm{~min} /$ week, an additional $100 \mathrm{~min} /$ week is prescribed, and for those meeting the target, an additional $50 \mathrm{~min} /$ week is prescribed. Printed material guiding participants to increase their activity level with worksheets is also provided. A diary in the format of a calendar returned monthly for 24 weeks is used to record PA and RPE to assess PA and intensity adherence. Participants may develop a medical problem or undergo treatment that can make exercising difficult or impossible. If this happens, prescribed amount of exercise is reviewed and represcribed, or stopped.

\section{Dietary session}

Participants at baseline who had unintentional weight loss of $5 \mathrm{~kg}$ or weight gain of $5 \mathrm{~kg}$ over the previous 6 months were seen by the dietitian. Furthermore, participants, whose diets at baseline scored low on one or more of the subscales of the ARFS (vegetables $<14$, fruit $<8$, protein $<4$, grains $<9$, dairy $<8$, water $<1),{ }^{39}$ were also seen by the dietitian. Dietary counselling was provided by a trained dietitian and overseen by the coordinating dietitian. During the 1-hour face-to-face counselling session, participants received individually tailored dietary advice and printed material explaining the diet in detail.

\section{Follow-ups}

Participants in the BBL-GP group are monitored through phone calls at weeks 4, 12 (14 for PA) and week 20 by the dietitian and exercise physiologist to monitor the progress and for reassurance. In addition, they receive a general booster session at 12 months with a phone call and a mailed-out booklet summarising materials from the online modules. They are being asked to continue being active and follow a healthy eating plan after completion of the intervention.

\section{Online modules}

Once participants in the BBL-GP group have received faceto-face PA and dietary counselling sessions, they are asked to $\log$ on to the trial website and complete one module per week, each taking approximately $30-40 \mathrm{~min}$. The 12-week programme is detailed in table 3 . The first 8 weeks include the completion of eight educational and individually tailored behaviour change modules. In the remaining 4 weeks, participants undertake online activities focused on goal monitoring and revision of the modules materials. Tailoring of the six behaviour change modules (weeks 3-8) is conducted using an automated algorithm that presents content on the basis of whether or not the participant has a relevant risk factor, as well as on the basis of their responses to several questions measuring psychological determinants of behaviour. These questions are presented at the beginning of each of the behaviour change modules. For instance, a person who is classified as having a poor diet (eg, lack of fish intake) on the basis of their responses on the ANU-ADRI, and who does not regard himself/herself as a role model to others with respect to their diet habits is not presented with information focusing on becoming a role model to others.

The programme is built in such a way that participants are only able to access the relevant component of the intervention at a given time. The modules become active, one per week, on the same day for the first 8 weeks. Participants are unable to access a newly activated module before completing the previously scheduled module. Each week, participants receive a notification email on the same day of the week alerting them when a new module has become active and a list of already activated modules that they have either not started or completed. Participants who are late completing modules will be followed up with an email from the project manager to identify if there is a reason (eg, holidays, illness, work commitments) preventing their participation, and encouraging them to continue with the study. Compliance is recorded for each module if they are completed on time, delayed or not completed. 
Table 3 Description of the 12-week online programme delivered through the Body Brain Life - General Practice (BBL-GP) website

\begin{tabular}{|c|c|c|}
\hline Week & Activity & Description \\
\hline 2 & $\begin{array}{l}\text { Module 2: dementia risk } \\
\text { factors }\end{array}$ & $\begin{array}{l}\text { This module is aimed at building awareness and knowledge of the various health } \\
\text { conditions associated with an increased risk of AD. Specifically, this module provides } \\
\text { details regarding the association between AD and several medical conditions (abnormal } \\
\text { weight, high cholesterol, diabetes, hypertension and depression), as well as lifestyle } \\
\text { factors (alcohol use and smoking, PA, nutrition, stroke and head injury, mental health, } \\
\text { social and cognitive engagement). The module also briefly covers non-modifiable risk } \\
\text { factors that contribute to AD, including age and genetics. }\end{array}$ \\
\hline 3 & Module 3: BBL PA & $\begin{array}{l}\text { This is a theory-driven, individually tailored module that aims to help participants } \\
\text { incorporate regular PA into their daily routine and reduce sedentary behaviour by } \\
\text { focusing on increasing endurance, strength, balance and flexibility. This module targets } \\
\text { several barriers to engaging in PA, such as increasing motivation, creating opportunities } \\
\text { to exercise and developing a social network that supports PA goals. }\end{array}$ \\
\hline 5 & $\begin{array}{l}\text { Module 5: BBL health self- } \\
\text { management }\end{array}$ & $\begin{array}{l}\text { This is a theory-driven, individually tailored module aimed at increasing participants' } \\
\text { health monitoring and management of chronic health conditions. Because several } \\
\text { chronic health conditions, such as hypertension, diabetes and high cholesterol, are } \\
\text { associated with increased risk for dementia, prevention and appropriate management of } \\
\text { such conditions are also likely to be protective against dementia. }\end{array}$ \\
\hline 6 & $\begin{array}{l}\text { Module 6: BBL think- } \\
\text { cognitive engagement }\end{array}$ & $\begin{array}{l}\text { This is a theory-driven, individually tailored module aimed at increasing participants' } \\
\text { levels of engagement with mentally stimulating activities such as reading, doing } \\
\text { crosswords and visiting museums, which is a protective factor against dementia. }\end{array}$ \\
\hline 9 & $\begin{array}{l}\text { 9-12 Self-guided online } \\
\text { activities }\end{array}$ & $\begin{array}{l}\text { During these sessions, participants are encouraged to engage in a range of online } \\
\text { activities for } 1 \text { hour, including accessing many tools they have accumulated during the } \\
\text { first } 8 \text { weeks. Examples include the goal-setting tool, behaviour-monitoring tool, unhelpful } \\
\text { thoughts monitoring tool, videos and so on. }\end{array}$ \\
\hline
\end{tabular}

AD, Alzheimer's disease; PA, physical activity.

Group 2: Lifestyle Modification Programme (LMP)

The Lifestyle Modification Programme (LMP), developed by NHC, is designed to provide individuals with tools to help manage chronic disease and maintain a healthy lifestyle. LMP is a 6-week group programme provided by various health professionals (dietitian, exercise physiologist, nurse practitioner, psychologist, pharmacist and sleep physician) providing information on basic nutrition, meal planning, PA, health conditions, motivation and goals, medications and sleep. Every week, two sessions are provided on the same day with each session lasting an hour. The course is currently run by the NHC for their patients to assist them in improving their lifestyle and management of chronic disease so it is a pragmatic real-life comparison condition. Attendance is recorded for compliance and motivation checking. Although the LMP is a free nationally recognised programme that is designed to provide individuals with tools to help manage chronic disease and maintain a healthy lifestyle, evaluation of the programme has not been carried out as yet. The attendance is recorded each week to examine intervention fidelity.

\section{Group 3: active control/email only}

The active control group or email-only group proceed with their normal activities and access health services as required over the trial period. Participants in this group also receive weekly emails containing links to various 
websites providing information on lifestyle risk factors and disease management for a duration of 12 weeks. The weekly emails contain several links, and participants are encouraged to spend approximately an hour each week browsing through the material. The material is generally organised around the same themes as the ones included in the BBL-GP Programme. An effort has been made to include links to relevant information and educational material, but that otherwise does not include the use of identifiable behaviour-change techniques which are the 'active ingredient' of the BBL-GP Programme. In addition, other than providing participants with the weekly emails, no further contact is made with the participants in this group, such as reminders and prompts that are provided to the BBL-GP group. Participants in this group receive a face-to-face, 1-hour risk-reduction workshop that provides the information contained in the BBL-GP intervention as a mean of debriefing at the end of the intervention.

\section{Masking}

To prevent performance bias, research staff conducting the assessments remain masked to participants' group allocation. The contact person for participants' website queries, access issues and technical difficulties is independent of all baseline assessment data. All participants are informed that they are being randomly allocated to one of three study groups and that one group may be more effective than others. They are also notified at the start of the study that one of these groups involves faceto-face group sessions which require them to travel to NHC head office. Hence, the research team members who recruit participants, conduct individual diet and PA sessions, and professionals who are involved in the LMP are naturally able to tell which group they have been allocated to. Nurses who conduct baseline and follow-up assessments are however masked to group allocation.

\section{Data management and monitoring}

A trial management committee is formed by the research team members (chief and co-investigators). Nursing staffs from NHC and research assistants collect, clean and send the study data to the committee on a weekly basis. Most data are automatically entered into excel files and other data are double entered to SPSS files to prevent data entry errors. Data management is then handled independently from the researchers who interpret the data. All data are stored electronically and in an independent spreadsheet and SPSS data file, which is only accessible by the researchers involved in this study.

An independent Data Monitoring Committee (DMC) is established independently from the research team who are involved with collecting and managing data. The DMC provides an independent oversight of the trial and reviews general conduct of the trial and study data for participant safety. The DMC comprises independent, multidisciplinary experts in dementia research who make recommendations regarding the continuation, modification or termination of the trial.

Adverse events (minor and serious) are monitored throughout the trial by the research team and any adverse events would be reported to the trial DMC. For this trial, an adverse event is defined as an unwanted and usually harmful outcome (eg, physical injuries). The event may or may not be related to the intervention, but it occurs while the person is participating in the intervention, that is, while they are undertaking physical activities individually prescribed by the exercise physiologist.

There are no formal interim analyses planned, as it is not expected that adverse events would be differentially related to the interventions.

\section{Statistical analyses}

Statistical analyses will be based on an intention-to-treat approach. As applied in the previous BBL project, ${ }^{15}$ multiple imputation and mixed models will be applied to analyse data. We hypothesise that the effectiveness of the intervention programmes will be in the following order: BBL-GP $>$ LMP $>$ active control. The hypothesis that BBL-GP will be more effective than LMP is based on research showing that a tailored programme is better than a one size fits all group programme in most cases. $^{48}$ This is also based on the previous BBL project where those in BBL groups improved more than those in the control group. We will also adjust for compliance in completing the online modules and following recommendations provided by the dietitian and exercise physiologist (for BBL-GP group), or for attendance to weekly group sessions (for LMP group).

\section{Adverse events}

This study evaluates lifestyle intervention programmes to reduce risk factors for AD. The target population is adults in a primary care setting who have some of the known risk factors for dementia, but are at the time of the intervention, healthy and free of any dementia-related symptoms. We do not anticipate that participants are placed at a greater risk than that associated with self-driven educational activities over the Internet. An adverse event where a participant can get hurt during prescribed exercise can occur. To prevent this, we screen participants using APSS at the baseline assessment to identify individuals with acute-risk/high-risk conditions for exercise. In addition, the exercise physiologist individually tailors prescribed exercise to minimise risk of injury. Medical assessments are done by the participants' usual nurses and doctors and if any abnormality is detected in their results, they are required to discuss these abnormalities with participants as usual. To address issues of potential fatigue, the assessments have been kept to a minimum length. In addition, all online and face-to-face sessions are designed in an interactive way and are limited to 1-hour sessions (LMP has two themed sessions per week and has a break between sessions). As mentioned above, all online modules are delivered in an 
individually tailored fashion to maximise relevance for each individual.

\section{Dissemination plan}

Positive, neutral and negative results of the trial will be submitted to international peer-reviewed journals. In addition, results will be presented at national and international conferences relevant to the subject matters. Authorship will be allocated using the guidelines for authorship defined by the International Committees of Medical Journal Editors and depends on personal involvement.

\section{DISCUSSION}

The project is currently under way as an evaluation of the efficacy of health promotion interventions in adults with risk factors for dementia. The programme aims to reduce cardiometabolic risk and promote behaviours shown to protect against dementia. The trial, recruiting from a primary care setting, has generated considerable interest, and to date, approximately half of the total target sample has been assessed and randomised into the intervention groups. We anticipate that all data collection will be completed by December 2018. The results of the study are likely to form an evidence base for the feasibility of dementia risk-reduction campaigns to lead to lifestyle changes and the reduction of dementia risk factors at the population level. This trial will also support the feasibility of such interventions being applied in primary care settings. Successful outcomes of the current trial may lead to significant public health impact and benefits once the intervention is made available at the population-level pending positive results.

\section{CONCLUSION}

Interventions to reduce risk of developing dementia are needed as a cure is not available. This project compares three different approaches to promote healthy lifestyles and to reduce risk of developing dementia applied in a primary care setting. This unique trial demonstrates reallife application of dementia risk-reduction intervention rather than more controlled but less ecologically valid interventions typically tested in a research setting.

\section{Author affiliations}

${ }^{1}$ Centre for Research on Ageing, Health and Wellbeing, Australian National University, Canberra, Australian Capital Territory, Australia

${ }^{2}$ School of Exercise and Nutrition Sciences, Deakin University, Burwood, Victoria, Australia

${ }^{3}$ Medical School, University of Western Australia, Crawley, Western Australia, Australia

${ }^{4}$ Academic Unit for Psychiatry of Old Age, University of Melbourne, Melbourne, Victoria, Australia

${ }^{5}$ Johns Hopkins Center on Aging and Health, Johns Hopkins University, Baltimore, Maryland, USA

${ }^{6}$ School of Medicine and Public Health, University of Newcastle, Newcastle, New South Wales, Australia

${ }^{7}$ National Centre for Epidemiology and Population Health (NCEPH), Australian National University, Canberra, Australian Capital Territory, Australia
${ }^{8}$ School of Psychology, University of New South Wales, Sydney, New South Wales, Australia

${ }^{9}$ Neuroscience Research Australia, Sydney, New South Wales, Australia

Acknowledgements The investigators acknowledge the work of staff at the National Health Co-op and research staff at the Centre for Research on Ageing, Health and Wellbeing. The investigators would particularly like to acknowledge the valued contribution of the trial participants.

Contributors KJA conceived the study and built relationship with NC. KJA, NC and SK developed the overall design of the intervention. NL and KLC designed the implementation of the PA-related intervention. $C D$ and KJA designed the statistical analysis plan for the protocol. DP and GWR contributed knowledge of primary care setting and advised on implementing the intervention in a GP clinic. ST designed the implementation of the dietary intervention. NC and SK managed the implementation of the online component in the dedicated web portal. SK, MM, ST, $\mathrm{KLC}, \mathrm{NL}, \mathrm{GWR}, \mathrm{DP}, \mathrm{IM}, \mathrm{NC}$ and KJA wrote the study protocol and critically reviewed the manuscript.

Funding This work was supported by National Health and Medical Research Council (NHMRC) Centre of Research Excellence in Cognitive Health and the development of original BBL modules was funded by the NHMRC Dementia Collaborative Research Centres. KJA is funded by NHMRC Fellowship APP1102694. Competing interests None declared.

Patient consent Not required.

Ethics approval The Human Research Ethics Committee at the Australian National University.

Provenance and peer review Not commissioned; externally peer reviewed.

Open Access This is an Open Access article distributed in accordance with the Creative Commons Attribution Non Commercial (CC BY-NC 4.0) license, which permits others to distribute, remix, adapt, build upon this work non-commercially, and license their derivative works on different terms, provided the original work is properly cited and the use is non-commercial. See: http://creativecommons.org/ licenses/by-nc/4.0/

(C) Article author(s) (or their employer(s) unless otherwise stated in the text of the article) 2018. All rights reserved. No commercial use is permitted unless otherwise expressly granted.

\section{REFERENCES}

1. Barnes DE, Yaffe K. The projected effect of risk factor reduction on Alzheimer's disease prevalence. Lancet Neurol 2011;10:819-28.

2. Ashby-Mitchell K, Burns R, Shaw J, et al. Proportion of dementia in Australia explained by common modifiable risk factors. Alzheimers Res Ther 2017:9:11.

3. Ngandu T, Lehtisalo J, Solomon A, et al. A 2 year multidomain intervention of diet, exercise, cognitive training, and vascular risk monitoring versus control to prevent cognitive decline in at-risk elderly people (FINGER): a randomised controlled trial. Lancet 2015;385:2255-63.

4. Prince M, Albanese E, Guerchet M, et al. World alzheimer report 2014: dementia and risk reduction. an analysis of protective and modifiable factors. London: ADI, 2014.

5. Santos CY, Snyder PJ, Wu WC, et al. Pathophysiologic relationship between Alzheimer's disease, cerebrovascular disease, and cardiovascular risk: a review and synthesis. Alzheimers Dement 2017;7:69-87.

6. Blondell SJ, Hammersley-Mather R, Veerman JL. Does physical activity prevent cognitive decline and dementia? A systematic review and meta-analysis of longitudinal studies. BMC Public Health 2014:14:510.

7. Northey JM, Cherbuin N, Pumpa KL, et al. Exercise interventions for cognitive function in adults older than 50: a systematic review with meta-analysis. Br J Sports Med 2018;52.

8. Mammen G, Faulkner G. Physical activity and the prevention of depression: a systematic review of prospective studies. Am J Prev Med 2013;45:649-57.

9. Brouwer BG, van der Graaf Y, Soedamah-Muthu SS, et al. Leisuretime physical activity and risk of type 2 diabetes in patients with established vascular disease or poorly controlled vascular risk factors. Diabetes Res Clin Pract 2010;87:372-8.

10. Middleton KR, Anton SD, Perri MG. Long-Term Adherence to Health Behavior Change. Am J Lifestyle Med 2013;7:395-404. 
11. Locke EA. Motivation through conscious goal setting. Applied and Preventive Psychology 1996;5:117-24.

12. The Department of Health. National primary health care strategic framework: Primary health care in Australia. $2013 \mathrm{http} / / \mathrm{www}$.health. gov.au/internet/publications/publishing.nsf/Content/NPHC-StrategicFramework phc-australia

13. Robinson L, Tang E, Taylor JP. Dementia: timely diagnosis and early intervention. BMJ 2015;350:h3029.

14. Moll van Charante EP, Richard E, Eurelings LS, et al. Effectiveness of a 6-year multidomain vascular care intervention to prevent dementia (preDIVA): a cluster-randomised controlled trial. Lancet 2016;388:797-805

15. Anstey KJ, Bahar-Fuchs A, Herath P, et al. Body brain life: a randomized controlled trial of an online dementia risk reduction intervention in middle-aged adults at risk of Alzheimer's disease. Alzheimers Dement 2015;1:72-80.

16. Boutron I, Moher D, Altman DG, et al. Extending the CONSORT statement to randomized trials of nonpharmacologic treatment: explanation and elaboration. Ann Intern Med 2008;148:295-309.

17. Zwarenstein M, Treweek S, Gagnier JJ, et al. Improving the reporting of pragmatic trials: an extension of the CONSORT statement. BMJ 2008;337:a2390.

18. Chan AW, Tetzlaff JM, Altman DG, et al. SPIRIT 2013 statement: defining standard protocol items for clinical trials. Ann Intern Med 2013;158:200-7.

19 Exercise \& Sports Science Australia. Adult pre-exercise screening system. $2011 \mathrm{https} / / / w w w . e s s a . o r g . a u / f o r-g p s / a d u l t-p r e-e x e r c i s e-$ screening-system/

20 Folstein MF, Folstein SE, McHugh PR. "Mini-mental state". A practical method for grading the cognitive state of patients for the clinician. J Psychiatr Res 1975;12:189-98.

21 Snell WE, Johnson G. The multidimensional health questionnaire. Am J Health Behav 1997;21:33-42.

22 Kim S, Cherbuin N, Anstey KJ. Assessing reliability of short and tick box forms of the ANU-ADRI: Convenient alternatives of a selfreport Alzheimer's disease risk assessment. Alzheimers Dement 2016;2:93-8.

23 Anstey KJ, Cherbuin N, Herath PM. Development of a new method for assessing global risk of Alzheimer's disease for use in population health approaches to prevention. Prev Sci 2013;14:411-21.

24. Anstey KJ, Cherbuin N, Herath PM, et al. A self-report risk index to predict occurrence of dementia in three independent cohorts of older adults: the ANU-ADRI. PLoS One 2014;9:e86141.

25. Colcombe S, Kramer AF. Fitness effects on the cognitive function of older adults: a meta-analytic study. Psychol Sci 2003;14:125-30.

26. Anstey KJ, Sargent-Cox K, Garde E, et al. Cognitive development over 8 years in midlife and its association with cardiovascular risk factors. Neuropsychology 2014;28:653-65.

27. Spreen O, Strauss E. A compendium of neuropsychological tests: administration, norms and commentary. New York: Oxford University Press, 1991.

28. Arbuthnott K, Frank J, Test TM. Trail making test, part B as a measure of executive control: validation using a set-switching paradigm. J Clin Exp Neuropsychol 2000;22:518-28.

29. Giovagnoli AR, Del Pesce M, Mascheroni S, et al. Trail making test: normative values from 287 normal adult controls. Ital J Neurol Sci 1996;17:305-9.
30. Smith A. Symbol digit modalities test: manual. Los Angeles: Western Psychological Services, 1982.

31. Craig CL, Marshall AL, Sjöström M, et al. International physical activity questionnaire: 12-country reliability and validity. Med Sci Sports Exerc 2003;35:1381-95.

32. Radloff L. The CES-D scale: a self report depression scale for research in the general population. Appl Psychol Meas 1977;1:385-401.

33. Ware J, Kosinski M, Keller SD. A 12-item short-form health survey: construction of scales and preliminary tests of reliability and validity. Med Care 1996;34:220-33.

34. Wilson PW, D'Agostino RB, Levy D, et al. Prediction of coronary heart disease using risk factor categories. Circulation 1998;97:1837-47.

35. Chen L, Magliano DJ, Balkau B, et al. AUSDRISK: an Australian type 2 diabetes risk assessment tool based on demographic, lifestyle and simple anthropometric measures. Med J Aust 2010;192:197-202.

36. Jakobsson U. Using the 12-item Short Form health survey (SF-12) to measure quality of life among older people. Aging Clin Exp Res 2007:19:457-64.

37. Maruish ME. User's manual for the SF-12v2 health survey. 3rd edn. Lincoln: Quality Metric Incorporated, 2012.

38. . Framingham Heart Study. 2017. https://www. framinghamheartstudy.org/risk-functions/coronary-heart-disease/ 10-year-risk.php

39. Collins CE, Burrows TL, Rollo ME, et al. The comparative validity and reproducibility of a diet quality index for adults: the Australian recommended food score. Nutrients 2015;7:785-98.

40. National Health and Medical Research Council. Australian dietary guidelines. Canberra: National Health and Medical Research Council, 2013.

41. National Health and Medical Research Council. Australian guide to healthy eating. Canberra: National Health and Medical Research Council, 2003.

42. Buysse DJ, Reynolds CF, Monk TH, et al. The Pittsburgh sleep quality index: a new instrument for psychiatric practice and research. Psychiatry Res 1989;28:193-213.

43. Backhaus J, Junghanns $\mathrm{K}$, Broocks $\mathrm{A}$, et al. Test-retest reliability and validity of the Pittsburgh Sleep Quality Index in primary insomnia. $J$ Psychosom Res 2002;53:737-40.

44. Anstey KJ, Bahar-Fuchs A, Herath P, et al. A 12-week multidomain intervention versus active control to reduce risk of Alzheimer's disease: study protocol for a randomized controlled trial. Trials 2013;14:60.

45. Lautenschlager NT, Cox KL, Flicker L, et al. Effect of physical activity on cognitive function in older adults at risk for Alzheimer disease: a randomized trial. JAMA 2008;300:1027-37.

46. Cyarto EV, Cox KL, Almeida OP, et al. The fitness for the Ageing Brain Study II (FABS II): protocol for a randomized controlled clinical trial evaluating the effect of physical activity on cognitive function in patients with Alzheimer's disease. Trials 2010;11:120.

47. Borg GA. Psychophysical bases of perceived exertion. Med Sci Sports Exerc 1982;14:377-81.

48. Kreuter MW, Oswald DL, Bull FC, et al. Are tailored health education materials always more effective than non-tailored materials? Health Educ Res 2000;15:305-15. 